\title{
Genetic correlation between autistic traits and $I Q$ in a population-based sample of twins with autism spectrum disorders (ASDs)
}

\author{
Takeshi Nishiyama ${ }^{1,2}$, Hitomi Taniai ${ }^{3}$, Taishi Miyachi ${ }^{4}$, Koken Ozaki $^{5}$, Makoto Tomita $^{6}$ and Satoshi Sumi ${ }^{7}$
}

Although there is accumulating evidence that intelligence quotient (IQ) indexes some aspects of the autistic spectrum disorders (ASDs), the causal relationship between autistic traits and IQ remains controversial. We examined the sources of covariation between autistic traits and IQ. As males have a four times greater risk of ASDs than females, gender-specific effects were also explored. Autistic traits and IQ were assessed in $\mathbf{4 5}$ twin male-male, female-female and opposite-sex pairs ascertained by the regional screening system in Nagoya, Japan. Sex-limited Cholesky structural equation models were used to decompose the correlations between autistic traits and IQ into genetic and environmental components, including sex-specific factors. Genetic correlations between autistic traits and IQ were high and not significantly different between boys and girls $(-0.94$ and -0.95 , respectively), but genetic factors underlying the autistic traits were not entirely shared with the IQ. The individual-specific environmental correlation between autistic traits and IQ was estimated at -0.29 for boys and -0.59 for girls. There is a substantial overlap between the genetic factors that influence individual variation in autistic traits and IQ, irrespective of gender. The individual life experiences that increase autistic traits, however, have a moderate overlap with those that contribute to individual IQs.

Journal of Human Genetics (2009) 54, 56-61; doi:10.1038/jhg.2008.3; published online 9 January 2009

Keywords: autistic spectrum disorders (ASDs); cholesky structural equation model; intelligence quotient (IQ); nosology; twin study

\section{INTRODUCTION}

It has been suggested that intelligence quotient (IQ) indexes some aspects of the autistic spectrum disorders (ASDs), which include autistic disorder $(\mathrm{AD})$, Asperger's disorder $(\mathrm{AsD})$ and pervasive developmental disorder not otherwise specified (PDDNOS) in the DSM-IV categories, by the empirical evidences regarding the natural history and clinical descriptions, including demographics, IQ profiles and comorbidity. ${ }^{1}$ For example, mental retardation (MR) is the most associated condition in ASDs, although conditions such as seizure, head circumference and other comorbid psychiatric symptoms are also notable., ${ }^{2,3}$ Approximately $50 \%$ of the children with ASDs are comorbid with MR, but this comorbidity rate increases to approximately $70 \%$ in children with $\mathrm{AD} .{ }^{4}$ In contrast, approximately $8 \%$ of the children with MR are comorbid with ASDs. ${ }^{5,6}$

There is evidence that autistic traits are highly heritable and are influenced by additive genetic and individual-specific environmental factors both in the general population ${ }^{7}$ and in the ASD population. ${ }^{8,9}$ In addition, there is evidence that IQ is fairly heritable and is influenced by additive genetic, shared environmental and individual- specific environmental factors both in the general population ${ }^{10}$ and in the mild MR population. ${ }^{11}$ However, the causal relationship between autistic traits and IQ remains controversial as some family studies of ASDs using standardized IQ tests found a higher rate of cognitive disabilities among siblings but others could not confirm this finding. In most studies of the parents of children with ASDs a cognitive impairment could not be established. ${ }^{12-16}$

An understanding of the extent to which autistic traits and IQ share genetic and environmental factors is important for classifying ASDs as well as for locating and identifying genes that contribute to ASDs. Surprisingly, there is no other published study on twins that assesses the relationship between autistic traits and IQ. In this study, we sought to determine the sources of individual covariation between the unidimensional autistic traits and IQ. Specifically, we examined to what extent both traits share the same genetic factors, environmental factors or both. In addition, we explored the potential sources of sex differences in these shared factors.

We use the term ASDs to refer to the DSM-IV category of pervasive developmental disorders (PDDs), except for Rett's disorder and

${ }^{1}$ Department of Information and Biological Sciences, Graduate School of Natural Sciences, Nagoya City University, Nagoya, Japan; ${ }^{2}$ Doctor of Public Health Program in Biostatistics, National Institute of Public Health, Wako, Japan; ${ }^{3}$ Department of Pediatrics, Nagoya Child Welfare Center, Nagoya, Japan; ${ }^{4}$ Osaka-Hamamatsu Joint Research Center for Child Mental Development, Hamamatsu, Japan; ${ }^{5}$ Japan Science and Technology Agency, Tokyo, Japan; ${ }^{6}$ Department of Mathematical Sciences, Nanzan University, Aichi, Japan and ${ }^{7}$ Department of Pediatrics, Nagoya Western Rehabilitation Center for Children with Disabilities, Nagoya, Japan

Correspondence: Dr T Nishiyama, Department of Information and Biological Sciences, Graduate School of Natural Sciences, Nagoya City University, Yamanohata 1, Mizuho-cho, Mizuho-ku, Nagoya 467-8601, Japan.

E-mail: nishiyama@minos.ocn.ne.jp

Received 21 August 2008; revised 27 October 2008; accepted 4 November 2008; published online 9 January 2009 
childhood disintegrative disorder, both of which are qualitatively different from $\mathrm{AD}, \mathrm{AsD}$ and PDDNOS in etiology and prognosis.

\section{METHODS}

\section{Subjects}

In this study, participants were a cohort of twins born between 1993 and 2004 who were ascertained through at least one proband to have ASDs. These twins were living in the northern, eastern and western regions of Nagoya city, Japan, which are administered by three district care centers for disabled children (Supplementary information 1). In this catchment area, all children with ASDs were ascertained through the regional screening system, which consists of a three-stage health checkup and also captures missed cases through referrals from kindergartens, nursery schools, clinics and hospital. Given that during the study the average participation rates were 93.2 and $87.5 \%$ for 18 -month-old and 3 -year-old children, respectively, and that more than $98 \%$ of the infants in the area attend kindergartens or nursery schools, most infants with developmental problems should have been noticed and thus the screening for ASDs could be considered to be thorough. If children are suspected of having ASDs, they are enrolled in a semi-structured group program or an individual therapy program held once a week at the centers. The details of the sample population have been described elsewhere. ${ }^{9,17}$

A consensus diagnosis of ASDs, based on the DSM-IV criteria, was made on the basis of all the information available in a semi-structured case vignette that was prepared based on medical examination, psychological assessment and a clinical report, through repeated observation, both by psychologists and by pediatric psychiatrists, of children between the ages of 4 and 6 years. Inter-rater reliability was assessed by studying 27 individuals, with their names, ages and zygosity information removed, and the kappa coefficient between the two raters (HT and MT) was 0.70 for $\mathrm{AD}$, AsD, PDDNOS and non-PDD.

In a total of 47 twin pairs, at least one proband was found to have ASDs. One monozygotic pair with twin-to-twin transfusion syndrome (TTTS) and one dizygotic pair in which a co-twin suffered from a cerebral palsy $(\mathrm{CP})$ were excluded. The reasons for exclusion were: (1) the recurrence risk of $\mathrm{CP}$ was much lower than that of MR; (2) serious prenatal and perinatal complications that are not necessarily genetic have been identified as etiologies for some children with $\mathrm{CP}^{18}$ and (3) TTTS is also a prenatal complication. The remaining 45 twin pairs were available for genetic analysis. The probandwise concordance rate (defined as the proportion of affected twin partners of probands) for ASDs was $97.3 \%$ for monozygote (MZ) pairs and $50.0 \%$ for dizygote (DZ) pairs (Table 1). All pairs were Japanese and were reared together.

Blind to diagnosis, the zygosity of the twins was determined by sex combination, placentation (a monochorionic twin pair could be diagnosed as an MZ pair; reverse not true), blood groups and the zygosity questionnaire administered to the twins' parents, which have been shown to be more than $90 \%$ accurate in distinguishing MZ from DZ twins. ${ }^{19}$ The determination of zygosity by combining all this information could be considered to be at least more accurate than that if only the questionnaire was used, although there remained the possibility that the potential misdiagnosis of the zygosity could confound the result.

Table 1 Twin pairs included in the study

\begin{tabular}{lcc}
\hline & Number of pairs & $\begin{array}{c}\text { Number of concordant pairs } \\
(\%)\end{array}$ \\
\hline MZM & 14 & $13(96.3)$ \\
MZF & 5 & $5(100)$ \\
MZ Total & 19 & $18(97.3)$ \\
DZM & 12 & $4(50.0)$ \\
DZF & 2 & $1(80.0)$ \\
DOS & 12 & $3(40.0)$ \\
DZ Total & 26 & $8(50.0)$ \\
\hline
\end{tabular}

Abbreviations: DOS, dizygotic opposite-sex pairs; DZF, dizygotic females; DZM, dizygotic males; MZF, monozygotic females; MZM, monozygotic males.

Concordance, the number of pairs concordant by ASDs (proband concordance rate \%; note that these are not pairwise concordance rates)

\section{Measures}

The Childhood Autism Rating Scale (CARS ${ }^{20}$ was used to assess the severity of autistic traits among ASDs as a unitary dimension. The CARS is a behaviorbased observer-rating scale with demonstrated reliability and validity in Japanese populations. ${ }^{21}$ The CARS comprises 15 items, which are rated from one to four, with a score of one indicating age-appropriate behavior and a score of four representing severely abnormal behavior. During a pilot period, 19 individuals were scored independently by two raters (TN and SS) who were trained extensively in CARS scoring and were blind to the individuals' names, ages, diagnoses and zygosity information. This inter-rater reliability analysis yielded an intraclass correlation coefficient of 0.91 (95\% CI; 0.78-0.96). Given this high reliability coefficient among the subsamples, only one of them scored the rest of CARS. IQ was also assessed using the Tanaka-Binet test (the standardized Japanese version of Stanford-Binet) by psychologists. As approved by the ethics committees in the facilities, and with parents providing written informed consent, both measures were administered to children between the ages of 4 and 6 years.

\section{Data analyses}

For autistic traits, we examined the internal consistency of the 15 items that make up the CARS by means of Cronbach's $\alpha$. Gender differences in the means of the CARS scores were tested using the $t$-statistic.

We applied a sex-limited bivariate Cholesky structural equation model $(\mathrm{SEM})^{22}$ to the twins' data to assess genetic and environmental factors shared between IQ and the autistic trait measured by CARS. The bivariate Cholesky model imposed a stratified structure on the latent factors hypothesized to determine the measured phenotypes, with one set of factors (additive genetic $(A)$, shared environmental $(C)$, individual-specific environmental $(E)$ ) influencing both an autistic trait and IQ, and the second set $(A, C, E)$ accounting for residual influences specific to IQ (Figure 1). This choice of ordering was based on the a priori hypothesis that an autistic trait may have specific risk factors over and above those shared with IQ because autistic traits have been shown to have higher heritability than IQ. In the SEM of twin studies, data from multiple samples are analyzed simultaneously, with some or all parameters constrained to be equal over the samples, where the samples represent different kinds of twin groups (MZ males, MZ females, DZ males, DZ females, DZ opposite-sex pairs). Therefore, we included all types of pairs in our analyses.

Sex-limitation models tested for two types of gender differences: (1) differences in the magnitude of effects of the same underlying latent (genetic and environmental) factors and (2) different sources of effects not shared between boys and girls (for example, sex-specific genes; $A_{F}$ in Figure 1). A re-parameterization of the bivariate Cholesky model in terms of correlations is

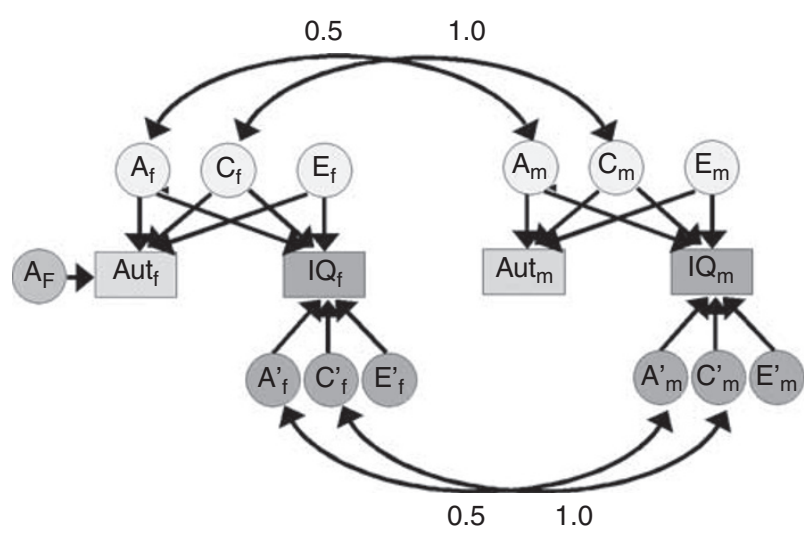

Figure 1 Sex-limitation Cholesky equation model for opposite-sex twin pair. Aut, autistic traits (CARS score); IQ, IQ test score; $A$, additive genetic influences; $C$, shared environmental influences; $E$, individual-specific environmental influences; $A_{\mathrm{F}}$, sex-specific genetic influences (in this case, female-specific). The subscripts $\mathrm{M}$ and $\mathrm{F}$ indicate the respective gender. $A$, $C$ and $E$ refer to factors common to both autistic traits and IQ. $A^{\prime}, C^{\prime}$ and $E^{\prime}$ refer to factors influencing only the IQ. 


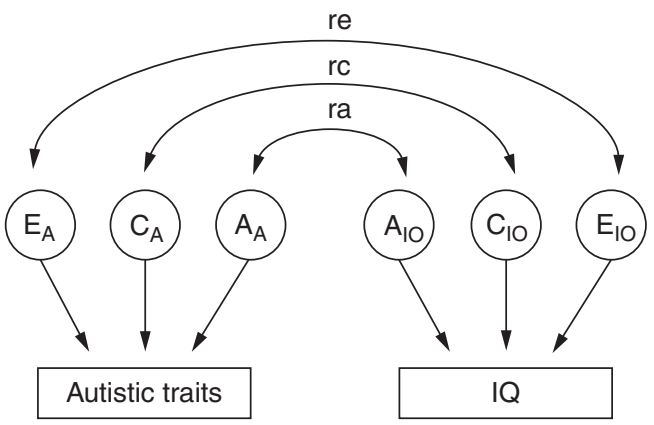

Figure 2 Bivariate twin model for autistic traits and IQ. The phenotypic correlation is decomposed into the additive genetic correlation $\left(r_{\mathrm{a}}\right)$ between additive genetic factors $\left(A_{\mathrm{A}}\right.$ and $\left.A_{I \mathrm{Q}}\right)$, the common environmental correlation $\left(r_{\mathrm{C}}\right)$ between common environmental factors $\left(C_{\mathrm{A}}\right.$ and $\left.C_{\mathrm{IQ}}\right)$, and the individualspecific environmental correlation $\left(r_{\mathrm{e}}\right)$ between individual-specific environmental factors $\left(E_{\mathrm{A}}\right.$ and $\left.E_{\mathrm{IQ}}\right)$ for autistic traits and $\mathrm{IQ}$, respectively.

schematically depicted in Figure 2 (for simplicity, only one twin pair is represented and sex-specific effects are not illustrated). In this model, the phenotypic correlation between an autistic trait and IQ in an individual was decomposed into the additive genetic $\left(r_{\mathrm{a}}\right)$, shared environmental $\left(r_{\mathrm{c}}\right)$ and individual-specific environmental $\left(r_{\mathrm{e}}\right)$ correlations between those latent factors.

We fit models to the raw data by using the Mx statistical package..$^{23}$ Model testing began with the full model that included all latent variables indicated in Figure 1. Model parameters and indices that characterized the fit of the model were calculated, and then this model was compared with nested submodels created by eliminating or constraining parameters in a stepwise fashion. The Akaike's information criterion (AIC) and the likelihood ratio $\chi^{2}$ test were used to compare nested models.

In addition, as we ascertained twin pairs through probands, an ascertainment correction was applied for the CARS and IQ score using the option for user-defined fit function in Mx script according to Wade et al. ${ }^{24}$ In brief, the likelihood of pairs not being in the ascertained sample can be expressed as:

$$
L_{\bar{A}}=\int_{-\infty}^{t} \int_{-\infty}^{\infty} \int_{-\infty}^{\infty} \int_{-\infty}^{\infty} \phi\left(x_{1}, y_{1}, x_{2}, y_{2} ; R\right) d x_{1} d y_{1} d x_{2} d y_{2}
$$

where $x_{1}$ and $x_{2}$ are variables for integration over the normal distribution of CARS score for a proband and his co-twin, $y_{1}$ and $y_{2}$ are variables of IQ score for a proband and his co-twin, $\phi$ is the four-variate normal probability density function, $R$ is the correlation matrix (transformed from the covariance matrix) and $t$ is the threshold used for ascertainment. Then, the likelihood of a pair of observations, $x_{1}$ and $x_{2}$, given the ascertainment scheme is:

$$
L\left(x_{1}, y_{1}, x_{2}, y_{2} \mid A\right)=\frac{\phi\left(x_{1}, y_{1}, x_{2}, y_{2} ; \Sigma\right)}{1-L_{\bar{A}}}
$$

where $\Sigma$ is the covariance matrix (determined by the variance components model; for more details, see Supplementary information 2). Ascertainment through probands can distort the distribution of the phenotypes of probands but not the conditional distribution of the phenotypes of co-twins given the phenotypes of probands. Therefore, for parameter estimation, it is appropriate to use this conditional distribution of the phenoypes of co-twins, given the phenotypes of probands.

The recently updated prevalence of ASDs, which is much higher than that reported earlier, is approximately $1 \%{ }^{25}$ Similarly, we also found that the prevalence of ASDs was $3.3 \%$ in males and $0.82 \%$ in females in our population, ${ }^{17}$ corresponding to the standard normal deviates of 1.84 and 2.40, respectively. These threshold values were used for ascertainment correction for males and for females.

All analyses, except as otherwise noted, were performed in the R statistical computing environment for Windows (Version 2.6.0). ${ }^{26}$ All tests conducted were two-tailed, with the type I error set at 0.05 .
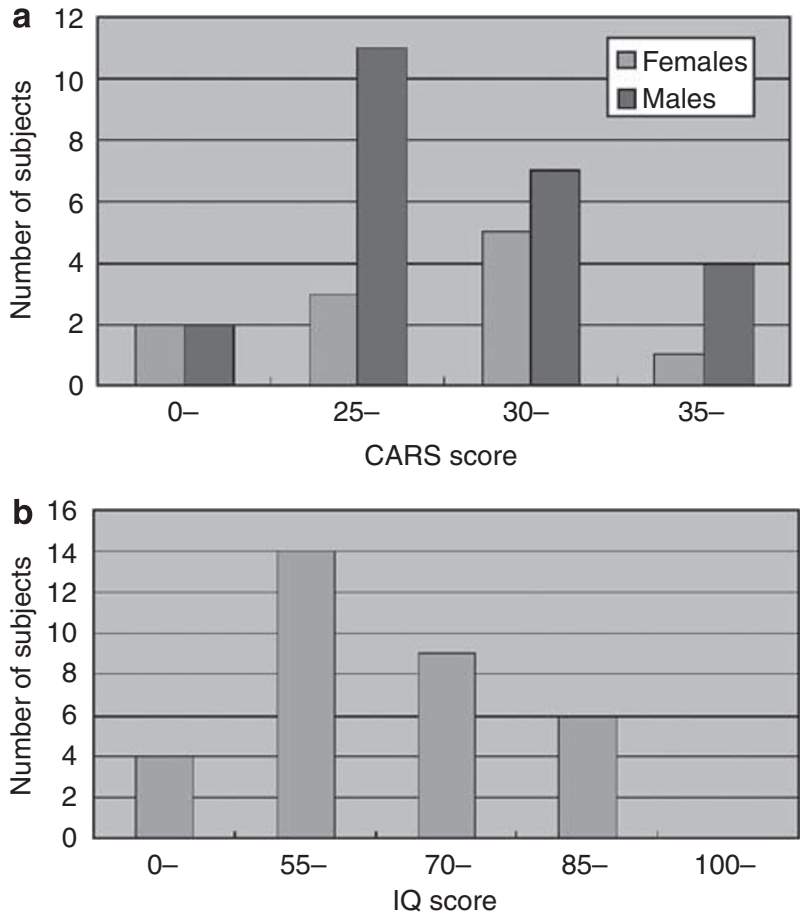

Figure 3 (a) Distribution of Child Autism Rating Scale (CARS) scores as a function of sex. (b) Distribution of intelligence quotient (IQ) scores.

\section{RESULTS}

The distribution of the CARS scores, plotted separately for boys and girls, is shown in Figure 3a. The mean \pm s.d. score for boys was $27.8 \pm 6.2$ and for girls was $26.7 \pm 7.0(t=0.74$, d.f. $=86, P=0.46$ with the pooled variance used). The distribution of IQ scores, plotted as a whole, is shown in Figure 3 b. The mean \pm s.d. score was $73.7 \pm 17.5$. Figure 3 shows that the distributions of both scores were not strongly skewed and there were no obvious nonlinear patterns in Q-Q plots (data not shown); therefore, raw scores were subjected to SEM for further analyses. The 15 items from CARS that make up the total score exhibited good internal consistency (Cronbach's $\alpha=0.93$ ). The correlations between the CARS and IQ score in twin pairs are given in Supplementary information 3.

Table 2 illustrates the results of our model-fitting procedure. The full model (model 1) included additive genetic, shared environmental and individual-specific environmental sources of variance and covariance for the CARS score and the IQ score, with both male-female shared and sex-specific genetic factors. Model 2 tested the significance of evidence for sex-specific genes by setting their path loading to zero. This resulted in a non-significant deterioration in fit and a more negative AIC compared with model $1(-2.00)$ and was, thus, more preferable. Based on our previous results of univariate model fitting, ${ }^{9}$ model 3 constrained the shared environmental factor in the CARS score and the shared environmental correlation between the CARS score and the IQ score to zero. This model was essentially identical in goodness of fit, but had more negative AIC (-9.42). Then, we constrained the magnitude of the additive genetic factors specific to the IQ score $\left(\mathrm{A}_{2}\right)$ to be equal across genders in model 4 . This model fit the data almost as well as model 3 , but with slightly more negative AIC, making it the overall best-fitting model by that criterion (Supplementary material 4). Model 5 constrained the genetic correlation between the CARS score and the IQ score to -1.0 as these are estimated to be near -1.0 in both sexes, forcing the assumption that 
Table 2 Fitting of bivariate sex-limitation Cholesky models of autistic traits and IQ to twin pairs ${ }^{\mathrm{a}}$

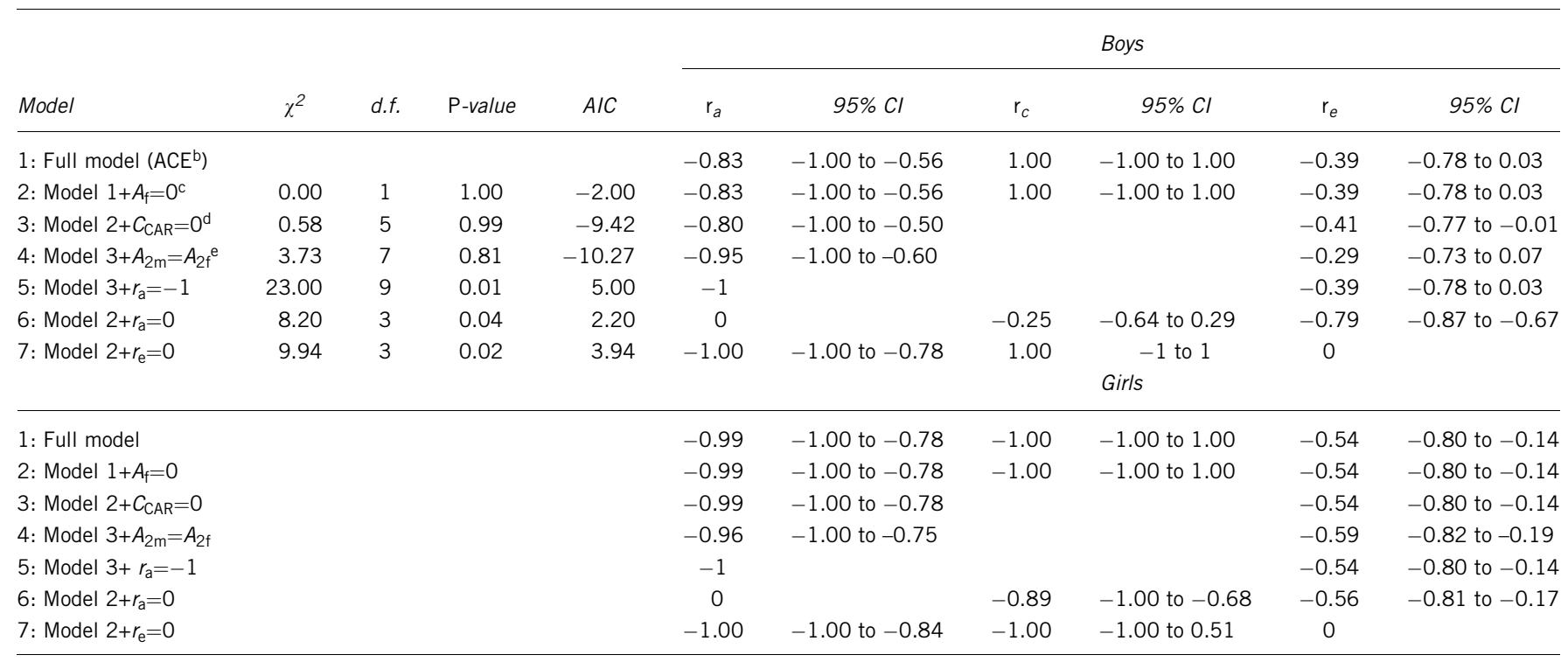

Abbreviations: AIC, Akaike's Information Criteria based on the full model; $\Delta$ d.f., degree of freedom.

$\chi^{2}$ and $\Delta$ d.f. are obtained by subtracting the fit function and d.f. of the full model from the fit function and the d.f. of interest.

${ }^{a} r_{a}$ indicates generic correlation; $r_{c}$, common environmental correlation; and $r_{\mathrm{e}}$, individual-specific environmental correlation between CARS score and IQ score.

${ }^{\mathrm{b}} A$ indicates additive genetic factors; $C$, common environmental factors; $E$, individual-specific environmental factors.

${ }^{c} A_{\mathrm{f}}=0$ indicates that sex-specific additive genetic factors were constrained to zero.

${ }^{\mathrm{d}} C_{\mathrm{CAR}}=0$ indicates that common environmental factors of CARS score were constrained to zero.

${ }^{\mathrm{e}} A_{2 \mathrm{~m}}=A_{2 \mathrm{f}}$ indicates that additive genetic factors specific to IQ score were constrained to be equal across genders.

the CARS score and the IQ score share all of their genes in common. This model resulted in a significantly poorer fit compared with model $1\left(\chi^{2}\right.$ Model $5=23.00, \Delta$ d.f. $\left.=9, P=0.006\right)$ and higher AIC (5.00). Models 6 and 7 tested the alternative hypotheses that the CARS score and the IQ score share none of their genes $\left(r_{\mathrm{a}}=0\right)$ or none of their individualspecific environmental factors $\left(r_{\mathrm{e}}=0\right)$ in common. Models 6 $\left(\chi^{2}\right.$ Model $6=8.204, \Delta$ d.f. $\left.=3, \quad P=0.042\right)$ and $7 \quad\left(\chi^{2}\right.$ Model $7=26.708$, $\Delta$ d.f. $=3, P<0.001)$ had a significantly poor fit to the data compared with the full model.

\section{DISCUSSION}

This study used bivariate modeling of twins' data to examine the genetic and environmental factors shared by autistic traits and IQ. Our results suggest that the additive genetic factors underlying autistic traits are not perfectly, but substantially, common to those that influence IQ. Individual-specific environmental factors, on the other hand, are modestly correlated between both traits. On applying the sex-limitation structure to these models, we found no evidence to support any sex-specific genetic factor. To our knowledge, this is the first study to examine the factors underlying the relationship between autistic traits and IQ. From our result suggesting substantial genetic correlation between autistic traits and IQ, it is predicted that there will be many genetic factors predisposing to ASDs as well as MR, and recently, copy number variations of chromosomes as such were actually identified. ${ }^{27,28}$ Regarding environmental factors, our results indicate that there are individual-specific environmental factors that influence both autistic traits and IQ, although most of the environmental factors that increase the autistic traits do not influence IQ and vice versa.

The results of this report should be interpreted in the context of several potential limitations. First, these findings largely depend on what instruments were used to measure autistic traits. For example, the Broader Phenotype Autism Symptom Scale (BPASS) and the CARS are moderately correlated to IQ, ${ }^{29,30}$ whereas the Social and

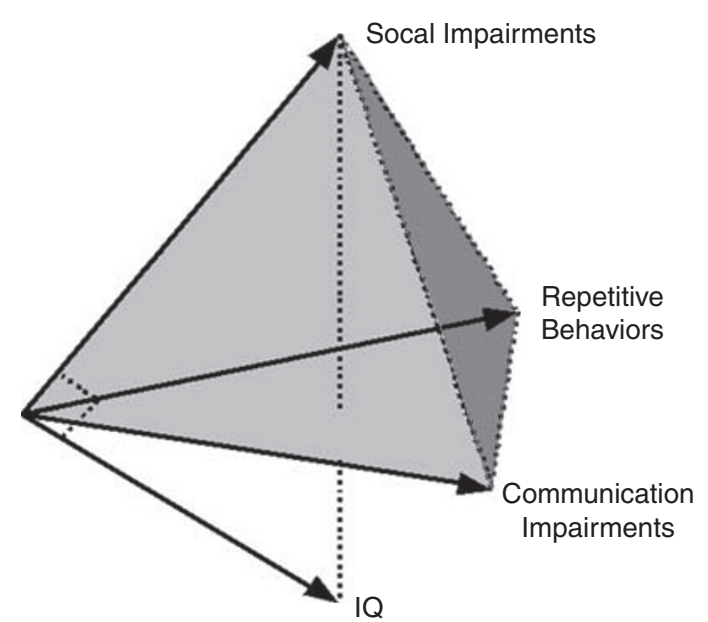

Figure 4 Conceptual model of the autism triad incorporating IQ. The dimension of social impairments is orthogonal to $I Q$, but the other two dimensions of communication impairments and repetitive behaviors are moderately correlated with IQ.

Communication Disorders Checklist (SCDC) and the Social Responsiveness Scale (SRS) are generally unrelated to IQ. ${ }^{31,32}$ These differences primarily reflect the characteristics of the instruments used. The SRS was specifically designed to tap social deficits that are inherent in ASD and the SCDC was also originally developed to measure social deficits in Turner's syndrome. In contrast, both the BPASS and CARS include broad-ranging domains of autistic symptomatology. Thus, social deficits seem to be independent of, but the other autistic traits, such as communication impairment and stereotypical behaviors, seem to be correlated to intellectual abilities (Figure 4). This finding would be supported, in part, by the studies suggesting that the type of repetitive behaviors in those with ASDs 
varied with $\mathrm{IQ}^{33,34}$ and also that communication impairment was associated with verbal abilities. ${ }^{35}$

Second, diagnosis of PDDNOS is problematic from a nosological point of view. The definition of PDDNOS has no explicit operational criteria, similar to all disorders with the 'NOS' qualifier. Severely disabled children with MR may, therefore, have autistic features but they might not be enough to warrant the diagnosis of $\mathrm{AD}$. This child, then, would receive the diagnosis of PDDNOS, indicating a mild degree of autism in the context of a severely handicapping condition. This phenomenon, called 'diagnostic substitution', can partially explain the growing identification of ASDs over the past decade. ${ }^{36}$ As children with PDDNOS outnumber those with AD by several-fold, the strong genetic correlation estimated in this study could be, in part, spurious because of an unreliable description of PDDNOS. In fact, the ability of experienced raters to differentiate reliably between $\mathrm{AD}$, AsD and PDDNOS, based on the DSM-IV criteria, has been found to be far from optimal. ${ }^{37}$

Third, the findings of this analysis are predicated based on the assumptions of SEM of twins. These assumptions include the unitarity of the observational variables, independence and additivity of the latent variables, absence of assortative mating and uniformity of environment, which imply that monozygotic and dizygotic twins are equally correlated in their exposure to environmental events. The CARS, one of the observational variables in the study, was designed to reflect the view that the varied symptoms shown by individuals with autism represented a unitary dimension. Indeed, the results of earlier reliable studies and those of this analysis indicated a high degree of internal consistency among the scale items of the CARS. Therefore, CARS score can be treated as a unitary variable with inter-rater reliability and discriminant validity. ${ }^{38}$ Another observational variable is IQ. In 4-year-old twins, genetic correlation between verbal and nonverbal cognitive abilities is approximately 0.6 both for the general population and for the low IQ extreme of the general population, ${ }^{39}$ and increases to approximately 1.0 in adolescence and adulthood. ${ }^{40}$ Therefore, IQ score can also be treated as unitary in genetic analysis. Although the presence of assortative mating has been suggested from the intergenerational study of autistic traits in the general population, ${ }^{7}$ we could not examine the presence of assortative mating because data regarding only one generation's twins were used. Similarly, other assumptions, such as the uniform environment assumption, could also not be tested in this study.

Fourth, although previous work suggests that estimates of the genetic contribution to variation in cognitive ability do not differ significantly between boys and girls, ${ }^{41}$ there might be a sex influence on the estimate of the genetic influence on IQ. It would be worthwhile to examine whether the estimate of the genetic correlation is different for boys and girls, which, however, would require a larger sample size.

Fifth, the relatively low sample size posed a significant challenge in model fitting. This lack of power is partly responsible for the wide confidence intervals estimated and may lead to imprecision in the estimation of parameters.

Finally, the results presented here are derived from a sample of twins. Although the twinning process itself is not a substantial risk factor in the etiology of autism, ${ }^{42}$ twins are known to have substantially lower IQ in childhood than singletons in the same family because of the shorter gestations and impaired fetal growth that affect some twins. ${ }^{43}$ This might affect generalization of the results to the singleton population, which is not subjected to experiences responsible for the cognitive delay in twins.

In conclusion, our results confirmed the existence of a substantial genetic association between autistic traits and IQ. Reliable and valid diagnostic procedures are a prerequisite for examining disease etiology. The diagnoses and classification of ASD have progressed considerably in the last 60 years, but much remains to be done for determining the role of IQ in classification of ASD.

\section{ACKNOWLEDGEMENTS}

There was no sponsor for this study. We thank Michael C Neale, $\mathrm{PhD}$, for assistance in model construction in $M x$ software. We also thank the families in the twin cohort for their participation.

1 Witwer, A. N. \& Lecavalier, L. Examining the validity of autism spectrum disorder subtypes. J. Autism Dev. Disord. 38, 1611-1624 (2008)

2 Miles, J. H., Hadden, L. L., Takahashi, T. N. \& Hillman, R. E. Head circumference is an independent clinical finding associated with autism. Am. J. Med. Genet. 95, 339-350 (2000).

3 Leyfer, O. T., Folstein, S. E., Bacalman, S., Davis, N. O., Dinh, E., Morgan, J. et al. Comorbid psychiatric disorders in children with autism: interview development and rates of disorders. J. Autism Dev. Disord. 36, 849-861 (2006).

4 Chakrabarti, S. \& Fombonne, E. Pervasive developmental disorders in preschool children: confirmation of high prevalence. Am. J. Psychiatry 162, 1133-1141 (2005).

5 Strømme, P. \& Diseth, T. H. Prevalence of psychiatric diagnoses in children with mental retardation: data from a population-based study. Dev. Med. Child Neurol. 42, 266-270 (2000).

6 Emerson, E. Prevalence of psychiatric disorders in children and adolescents with and without intellectual disability. J. Intellect. Disabil. Res. 47, 51-58 (2003).

7 Constantino, J. N. \& Todd, R. D. Intergenerational transmission of subthreshold autistic traits in the general population. Biol. Psychiatry 57, 655-660 (2005).

8 Bailey, A., Le Couteur, A., Gottesman, I., Bolton, P., Simonoff, E., Yuzda, E. et al. Autism as a strongly genetic disorder: evidence from a British twin study. Psychol. Med. 25, 63-77 (1995)

9 Taniai, H., Nishiyama, T., Miyachi, T., Imaeda, M. \& Sumi, S. Genetic influences on the broad spectrum of autism: study of proband-ascertained twins. Am. J. Med. Genet. B. Neuropsychiatr. Genet. 147B, 844-849 (2008).

10 Spinath, F. M., Ronald, A., Harlaar, N., Price, T. S. \& Plomin, R Phenotypic g early in life: On the etiology of general cognitive ability in a large population sample of twin children aged 2-4 years. Intelligence 31, 195-210 (2003).

11 Spinath, F. M., Harlaar, N., Ronald, A. \& Plomin, R. Substantial genetic influence on mild mental impairment in early childhood. Am. J. Ment. Retard 109, 34-43 (2004).

12 Fombonne, E., Bolton, P., Prior, J., Jordan, H. \& Rutter, M. A family study of autism: cognitive patterns and levels in parents and siblings. J. Child Psychol. Psychiatry 38, 667-683 (1997).

13 Folstein, S. E., Santangelo, S. L., Gilman, S. E., Piven, J., Landa, R., Lainhart, J. et al. Predictors of cognitive test patterns in autism families. J. Child. Psychol. Psychiatry 40, 1117-1128 (1999).

14 Spiker, D., Lotspeich, L. J., Dimiceli, S., Myers, R. M. \& Risch, N. Behavioral phenotypic variation in autism multiplex families: evidence for a continuous severity gradient. Am. J. Med. Genet. 114, 129-136 (2002).

15 MacLean, J. E., Szatmari, P., Jones, M. B., Bryson, S. E., Mahoney, W. J., Bartolucci, G. et al. Familial factors influence level of functioning in pervasive developmental disorder. J. Am. Acad. Child. Adolesc. Psychiatry 38, 746-753 (1999).

16 Goin-Kochel, R. P., Mazefsky, C. A. \& Riley, B. P. Level of functioning in autism spectrum disorders: phenotypic congruence among affected siblings. J. Autism. Dev. Disord 38, 1019-1027 (2008).

17 Sumi, S., Taniai, H., Miyachi, T. \& Tanemura, M. Sibling risk of pervasive developmental disorder estimated by means of an epidemiologic survey in Nagoya, Japan. J. Hum. Genet. 51, 518-522 (2006).

18 Van Naarden Braun, K., Autry, A. \& Boyle, C. A population-based study of the recurrence of developmental disabilities-Metropolitan Atlanta Developmental Disabilities Surveillance Program, 1991-94. Paediatr. Perinat. Epidemiol. 19, 69-79 (2005).

19 Ooki, S. \& Asaka, A. Zygosity diagnosis in young twins by questionnaire for twins 'mothers and twins' self-reports. Twin Res 7, 5-12 (2004).

20 Schopler, E., Reichler, R. J., DeVellis, R. F. \& Daly, K. Toward objective classification of childhood autism: Childhood Autism Rating Scale (CARS). J. Autism Dev. Disord 10, 91-103 (1980).

21 Kurita, H., Miyake, Y. \& Katsuno, K. Reliability and validity of the childhood autism rating scale-Tokyo version (CARS-TV). J. Autism Dev. Disord 19, 389-396 (1989).

22 Neale, M. C. \& Cardon, L. R. Methodology for Genetics Studies of Twins and Families (Kluwer Academic Publishers: Boston, 1992).

23 Neale, M. C. Mx: Statistical Modeling, 5th edn. (Richmond, Virginia, 1999).

24 Wade, T., Neale, M. C., Lake, R. I. \& Martin, N. G. A genetic analysis of the eating and attitudes associated with bulimia nervosa: dealing with the problem of ascertainment in twin studies. Behav. Genet 29, 1-10 (1999).

25 Baird, G., Simonoff, E., Pickles, A., Chandler, S., Loucas, T., Meldrum, D. et al. Prevalence of disorders of the autism spectrum in a population cohort of children in 
South Thames: the Special Needs and Autism Project (SNAP). Lancet 368, 210-215 (2006).

$26 \mathrm{R}$ Development Core Team. R: A language and environment for statistical computing (R Foundation for Statistical Computing: Vienna, Austria, 2004).

27 Marshall, C. R., Noor, A., Vincent, J. B., Lionel, A. C., Feuk, L., Skaug, J. et al. Structural variation of chromosomes in autism spectrum disorder. Am. J. Hum. Genet 82, 477-488 (2008).

28 Sebat, J., Lakshmi, B., Malhotra, D., Troge, J., Lese-Martin, C., Walsh, T. et al. Strong association of de novo copy number mutations with autism. Science 316, 445-449 (2007).

29 Perry, A., Condillac, R. A., Freeman, N. L., Dunn-Geier, J. \& Belair, J. Multi-site study of the Childhood Autism Rating Scale (CARS) in five clinical groups of young children. J. Autism Dev. Disord 35, 625-634 (2005).

30 Dawson, G., Estes, A., Munson, J., Schellenberg, G., Bernier, R. \& Abbott, R. Quantitative assessment of autism symptom-related traits in probands and parents: Broader Phenotype Autism Symptom Scale. J. Autism Dev. Disord 37, 523-536 (2007).

31 Constantino, J. N., Davis, S. A., Todd, R. D., Schindler, M. K., Gross, M. M., Brophy, S. L. et al. Validation of a brief quantitative measure of autistic traits: comparison of the social responsiveness scale with the autism diagnostic interview-revised. J. Autism Dev. Disord. 33, 427-433 (2003).

32 Skuse, D. H., Mandy, W. P. \& Scourfield, J. Measuring autistic traits: heritability, reliability and validity of the Social and Communication Disorders Checklist. Br. J. Psychiatry 187, 568-572 (2005).

33 Richler, J., Bishop, S. L., Kleinke, J. R. \& Lord, C. Restricted and repetitive behaviors in young children with autism spectrum disorders. J. Autism Dev. Disord 37, 73-85 (2007).
34 Hus, V., Pickles, A., Cook\#\#Jr, E. H., Risi, S. \& Lord, C. Using the autism diagnostic interview-revised to increase phenotypic homogeneity in genetic studies of autism. Biol. Psychiatry 61, 438-448 (2007).

35 Joseph, R. M., Tager-Flusberg, H. \& Lord, C. Cognitive profiles and social-communicative functioning in children with autism spectrum disorder. J. Child Psychol. Psychiatry 43, 807-821 (2002).

36 Shattuck, P. T. The contribution of diagnostic substitution to the growing administrative prevalence of autism in US special education. Pediatrics 117, 1028-1037 (2006).

37 Mahoney, W. J., Szatmari, P., MacLean, J. E., Bryson, S. E., Bartolucci, G., Walter, S. D. et al. Reliability and accuracy of differentiating pervasive developmental disorder subtypes. J. Am. Acad. Child Adolesc. Psychiatry 37, 278-285 (1998).

38 Tachimori, H., Osada, H. \& Kurita, H. Childhood autism rating scale-Tokyo version for screening pervasive developmental disorders. Psychiatry Clin. Neurosci 57, 113-118 (2003).

39 Price, T. S., Dale, P. S. \& Plomin, R. A longitudinal genetic analysis of low verbal and nonverbal cognitive abilities in early childhood. Twin Res 7, 139-148 (2004).

40 Casto, S. D., DeFries, J. C. \& Fulker, D. W. Multivariate genetic analysis of Wechsler Intelligence Scale for Children-Revised (WISC-R) factors. Behav Genet 25, 25-32 (1995).

41 Knopik, V. S. \& DeFries, J. C. A twin study of gender-influenced individual differences in general cognitive ability. Intelligence 26, 81-89 (1998).

42 Hallmayer, J., Glasson, E. J., Bower, C., Petterson, B., Croen, L., Grether, J. et al. On the twin risk in autism. Am. J. Hum. Genet 71, 941-946 (2002).

43 Ronalds, G. A., De Stavola, B. L. \& Leon, D. A. The cognitive cost of being a twin: evidence from comparisons within families in the Aberdeen children of the 1950s cohort study. B.M.J. 331, 1306-1310 (2005).

Supplementary Information accompanies the paper on Journal of Human Genetics website (http://www.nature.com/jhg) 